Pacific Journal of Mathematics

A REMARK ON FIELDS WITH THE DENSE ORBITS
PROPERTY 


\title{
A REMARK ON FIELDS WITH THE DENSE ORBITS PROPERTY
}

\author{
JEsÚs M. RUIZ
}

Let $K$ be a formally real field and $\Omega$ its order space. The automorphisms group of $K$ acts on $\Omega$, and $K$ is called D.O.P. when all the orbits are dense in $\Omega$. In this note the following is shown: The field of meromorphic function germs of a real irreducible analytic germ of dimension $>1$ is never D.O.P.

A formally real field $K$ has an associated order space $\Omega=\operatorname{Spec}_{R} K$. The automorphisms group $\operatorname{Aut}(K)$ of $K$ acts over $\Omega$ in an obvious way, and the space of orbits under this action measures the homogeneity of $\Omega$. Actually, $\Omega$ or $K$ are called homogeneous if $\operatorname{Aut}(K)$ acts transitively. There is also a weaker homogeneity condition, the so-called "dense orbits property": $K$ is D.O.P. if all orbits are dense in $\Omega$ (cf. [2], [4]). These notions are specially considered in the geometric case, i.e., when $K$ is a field of functions. It turns out then that both are very strong rigidity conditions. Indeed, let $V$ be an irreducible algebraic variety over $\mathbf{R}$ and $K$ its field of rational functions:

(a) If $K$ is homogeneous, then $V$ is a curve, either rational or elliptic.

(b) If $\operatorname{dim} V=1$ and $K$ is D.O.P., then $V$ is homogeneous.

(c) If $V$ is of general type, then $K$ is not D.O.P.

(These results are contained in [3], although in some slightly different form.) Thus, the open problem is to characterize D.O.P. (fields of functions of) varieties. Some partial answers are known (for instance, affine spaces are D.O.P., cf. [2]), but the general solution appears to be quite misterious. In this small note we show that this is certainly not the case in a local context. i.e., for analytic germs. Namely, we prove:

THEOREM. Let $X_{0}$ be an irreducible analytic germ in $\mathbf{R}_{0}^{n}$ and $K$ its field of real meromorphic function germs. If $\operatorname{dim} X_{0}>1, K$ is not D.O.P.

REMARK. If $\operatorname{dim} X_{0}=1$, then $K$ is nothing but the quotient field of $\mathbf{R}\{t\}$ and it is trivially homogeneous. The interesting question in this case appears to be whether $\Omega$ is still homogeneous under the action of $\operatorname{Aut}(A)$, $A$ being the ring of analytic function germs in $X_{0}$. 
2. Proof of the theorem. We may clearly assume that $X_{0}$ is normal. First we need the following

(2.1) Lemma. Any $\psi \in \operatorname{Aut}(K)$ is induced by some (unique) analytic diffeomorphism $\psi^{*}$ of $X_{0}$.

Proof. Let $A$ stand for the ring of analytic function germs on $X_{0}$, so that $K=q f A$. We have to check that $\psi(A) \subset A$ and $\psi \mid \mathbf{R}=\operatorname{Id}_{\mathbf{R}}$. Let $h \in A, h \neq 0$ and $h(0) \neq 1$. Set $\psi(h)=x \in K$. For each $n \geq 0$ there is $h_{n}=(1-h)^{1 / n} \in A$ (implicit function theorem) and, setting $y_{n}=\psi\left(h_{n}\right)$, we have: $\left(y_{n}\right)^{n}=1-x=y$. Now consider any discrete valuation $\nu$ of $K$ over $A$. As $\nu(y)=n \nu\left(y_{n}\right)$, we conclude $\nu(y)=0$ (were $\nu(y) \neq 0$, would it be $|\nu(y)|=n\left|\nu\left(y_{n}\right)\right| \geq n$, all $\left.n\right)$. Thus, $y \in \bigcap_{\nu} A_{\nu}=A, A$ being normal, and $x \in A$.

This proves $\psi(A) \subset A$ and, by symmetry, $\psi(A)=A$. Hence $\psi$ : $A \rightarrow A$ is an isomorphism and in particular a local homomorphism of analytic rings. But by a result in [1], this implies $\psi \mid \mathbf{R}=\mathrm{Id}_{\mathbf{R}}$, and the proof is complete.

EXAMPLE. If $X_{0}$ is not normal, 2.1 is not true any more: take $A=\mathbf{R}\left\{t^{2}, t^{5}\right\} \subset \mathbf{R}\{t\} \subset K$ and $\psi$ induced by $t \mapsto t+t^{2}$.

We now prove our theorem.

Step I. Let $X_{0}^{*}$ denote the maximum dimension locus of $X_{0}$. There are two analytic half-branches $c_{0}$ and $c_{0}^{\prime}$, both in $X_{0}^{*}$, whose multiplicities $m$ and $m^{\prime}$ are different, say $m<m^{\prime}$.

Indeed, after a standard application of local parametrization the problem reduces to find $c_{0}$ with arbitrarily large $m$ in some given non-empty open semianalytic germ $W_{0} \subset \mathbf{R}_{0}^{d}$ and, after a new linear projection, $d=2$ (notice that transversal projections do not increase multiplicities). Also, we can suppose

$$
W_{0}=\left\{(x, y) \in \mathbf{R}^{2}: x>0, h\left(x^{1 / p}\right)<y<g\left(x^{1 / p}\right)\right\}
$$

for some $p \geq 1, h, g \in \mathbf{R}\{t\}$. As $W_{0} \neq \varnothing$ we can write:

$$
h=h_{0}(t)+t^{r} h_{1}(t), \quad g=g_{0}(t)+t^{r} g_{1}(t),
$$

where $h_{0}(t), g_{0}(t)$ are polynomials in $\mathbf{R}[t]$ of degree $<r$ and $h_{0}<g_{0}$ for small $t$. Then choose $f_{0} \in \mathbf{R}[t]$, of degree $<r$, such that $h_{0}<f_{0}<g_{0}$ for small $t$. It follows that for any Puiseux series $f_{1}\left(t^{1 / q}\right)$ the curve germ

$$
t \mapsto\left(t^{p q}, f_{0}\left(t^{q}\right)+t^{q r} f_{1}(t)\right), \quad t>0
$$


is contained in $W_{0}$, and if $f_{1}$ and $q$ are well chosen, the multiplicity of this curve germ is arbitrarily large. This completes Step I.

Step II. There is an open semianalytic germ $U_{0} \subset \mathbf{R}_{0}^{n}$, such that $c_{0}^{\prime} \subset U_{0}$, and any curve germ $c_{0}^{*} \subset U_{0}$ has multiplicity $m^{*} \geq m^{\prime}$.

For, like in Step I, it is enough to consider the case $n=2$, and $c_{0}^{\prime}$ given by: $y=h\left(x^{1 / m^{\prime}}\right), x>0$, where $h \in \mathbf{R}\{t\}$. We write $h$ in the following way:

$$
h=a_{1} t^{p_{1}}+\cdots+a_{r} t^{p_{r}}+a t^{p}+t^{p+1} h_{1}(t), \quad 1 \leq p_{1}<\cdots<p_{r}<p,
$$

and $\left(p_{1}, \ldots, p_{r}, m^{\prime}\right)=1$. Then the $U_{0}$ we sought is

$$
\left\{x>0, h^{-}\left(x^{1 / m^{\prime}}\right)<y<h^{+}\left(x^{1 / m^{\prime}}\right)\right\}
$$

where

$$
\begin{aligned}
& h^{-}(t)=a_{1} t^{p_{1}}+\cdots+a_{r} t^{p_{r}}+(a-\varepsilon) t^{p}, \\
& h^{+}(t)=a_{1} t^{p_{1}}+\cdots+a_{r} t^{p_{r}}+(a+\varepsilon) t^{p}
\end{aligned}
$$

(pick any $\varepsilon>0$ ). Indeed, if $c_{0}^{*} \subset U_{0}$ is given by $y=f\left(x^{1 / m^{*}}\right), x>0$, we have

$$
h^{-}\left(x^{1 / m^{\prime}}\right)<f\left(x^{1 / m^{*}}\right)<h^{+}\left(x^{1 / m^{\prime}}\right) \quad(\text { for small } x>0)
$$

and so

$$
f\left(x^{1 / m^{*}}\right)=a_{1} x^{p_{1} / m^{\prime}}+\cdots+a_{r} x^{p_{r} / m^{\prime}}+f_{1}\left(x^{1 / m^{*}}\right) .
$$

But $m^{*}$ is the lowest common denominator of all exponents in this development, so $m^{*} \geq m^{\prime}, m^{\prime}$ being the 1.c.d. of the first $r$ exponents. Thus we are done.

Step III: end of the proof. By the separation lemma in [6], there is an analytic function germ $h$ such that $c_{0}^{\prime} \subset\{h>0\} \subset\{h \geq 0\} \backslash\{0\} \subset U_{0}$. Let $\alpha^{\prime}$ be any ordering centered at $c_{0}^{\prime}$ (such an $\alpha^{\prime}$ does exist, as $c_{0}^{\prime} \subset X_{0}^{*}$, cf. [5]) and consider the open neighborhood $H=H(h)$ of $\alpha^{\prime}$ in $\Omega=$ $\operatorname{Spec}_{R} K$. We claim that $H$ contains no ordering $\alpha^{*}$ isomorphic to any ordering $\alpha$ centered at $c_{0} \subset X_{0}^{*}$, what finishes the proof (again because $\left.c_{0} \subset X_{0}^{*}\right)$. Indeed, assume the contrary, i.e. there is $\psi \in \operatorname{Aut}(K)$ such that $\psi \alpha \in H$. Then by Lemma 2.1, we get an analytic half-branch $\psi^{*-1} c_{0}=c_{0}^{*}$. As $h$ must be positive in $\psi \alpha, c_{0}^{*} \subset\{h \geq 0\} \backslash\{0\} \subset U_{0}$ and mult $c_{0}^{*}=$ $m^{*} \geq m^{\prime}$, because of the choice of $U_{0}$ (Step II). But $c_{0}^{*}$ is analytically diffeomorphic to $c_{0}$, hence $m^{*}=m<m^{\prime}$, contradiction. 
REMARK. One technical point in the proof above is to be sure $c_{0}^{*}$ is within $U_{0}$ and not merely in the boundary. It is here where the separation lemma is useful. Yet there is another possibility: using formal half-branches (cf. [5]). But then one needs an approximation lemma for these halfbranches (loc. cit.) and the proof becomes more involved than it actually is.

\section{REFERENCES}

[1] S. S. Abhyankar and M. van der Put, Homomorphisms of analytic local rings, J. Reine Angew Math., 242 (1970), 27-33.

[2] D. W. Dubois and T. Recio, Order extensions and real algebraic geometry, in Amer. Math. Soc. Contemporary Math., 8 (1981).

[3] J. M. Gamboa, A characterization of rational and elliptic curves in terms of their space of orderings, Rocky Mountain J. Math., 14 (1984), 499-502.

[4] J. M. Gamboa and T. Recio, Ordered fields with the dense orbits property, J. Pure Appl. Algebra, 30 (1983), 237-246.

[5] J. M. Ruiz, Central orderings in fields of meromorphic function germs, Manuscripta Math., 46 (1984), 193-214.

[6] __ A note on a separation problem, Arch. Math., 43 (1984), 422-426.

Received June 20, 1984.

UNIVERSIDAD COMPLUTENSE DE MADRID

MADRID (3), SPAIN 


\section{PACIFIC JOURNAL OF MATHEMATICS EDITORS}

V. S. VARADARAJAN (Managing Editor)
University of California
Los Angeles, CA 90024
HEBERT CLEMENS
University of Utah
Salt Lake City, UT 84112
CHARLES R. DEPRIMA
California Institute of Technology
Pasadena, CA 91125

\section{R. FINN}

Stanford University

Stanford, CA 94305

HeRmanN FlaschKa

University of Arizona

Tucson, AZ 85721

RAMESH A. GANGOLli

University of Washington

Seattle, WA 98195

ROBION KIRBY

University of California

Berkeley, CA 94720

\section{C. MoOre}

University of California

Berkeley, CA 94720

H. SAMELSON

Stanford University

Stanford, CA 94305

HAROLD STARK

University of California, San Diego

La Jolla, CA 92093

ASSOCIATE EDITORS
R. ARENS
E. F. BECKENBACH
B. H. NeUmanN
F. WOLF
K. Yoshida (1906-1982)

\section{SUPPORTING INSTITUTIONS}

UNIVERSITY OF ARIZONA

UNIVERSITY OF BRITISH COLUMBIA

CALIFORNIA INSTITUTE OF TECHNOLOGY

UNIVERSITY OF CALIFORNIA

MONTANA STATE UNIVERSITY

UNIVERSITY OF NEVADA, RENO

NEW MEXICO STATE UNIVERSITY

OREGON STATE UNIVERSITY
UNIVERSITY OF OREGON

UNIVERSITY OF SOUTHERN CALIFORNIA

STANFORD UNIVERSITY

UNIVERSITY OF HAWAII

UNIVERSITY OF TOKYO

UNIVERSITY OF UTAH

WASHINGTON STATE UNIVERSITY

UNIVERSITY OF WASHINGTON 


\section{Pacific Journal of Mathematics}

\section{Vol. 121, No. $1 \quad$ November, 1986}

Om P. Agrawal, Douglas Napier Clark and Ronald George Douglas,

Invariant subspaces in the polydisk $\ldots \ldots \ldots \ldots \ldots \ldots \ldots \ldots \ldots \ldots$

Christoph Bandt and Gebreselassie Baraki, Metrically invariant measures

on locally homogeneous spaces and hyperspaces $\ldots \ldots \ldots \ldots \ldots \ldots \ldots$

Marcy Mason Barge, Horseshoe maps and inverse limits ..............29

Russell Gene Bilyeu, Robert Richard Kallman and Paul Weldon Lewis,

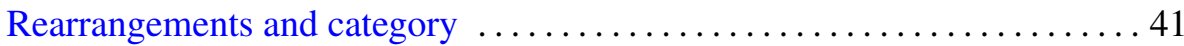

Jean Bourgain, A problem of Douglas and Rudin on factorization . . .......47

Hernan Cendra, A normal form and integration in finite terms for a class of

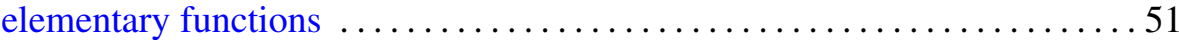

Ky Fan, The angular derivative of an operator-valued analytic function . . . . 67

Gerhard Gierz, On the Dunford-Pettis property of function modules of

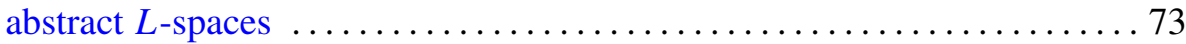

Gabriel Katz, On polynomial generators in the algebra of complex functions

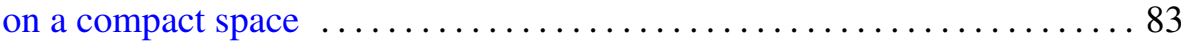

Ridgley Lange, Duality and asymptotic spectral decompositions $\ldots \ldots \ldots . .93$

Anthony To-Ming Lau and Peter F. Mah, Quasinormal structures for certain spaces of operators on a Hilbert space ................... 109

R. Daniel Mauldin, Correction: "The set of continuous nowhere differentiable functions"

Alan Harvey Mekler and Saharon Shelah, $\omega$-elongations and Crawley's problem

Alan Harvey Mekler and Saharon Shelah, The solution to Crawley's problem

Richard Rochberg, Deformation of uniform algebras on Riemann surfaces

Joseph Roitberg, On weak epimorphisms in homotopy theory

Jesús M. Ruiz, A remark on fields with the dense orbits property

Henry Wente, Counterexample to a conjecture of H. Hopf

David G. Wright, Rigid sets in $E^{n}$ 